the controls. Antisocial personality disorder (12\% versus $3 \%$ ) and nonalcohol substance abuse $(12 \%$ versus $4 \%)$ were significantly more prevalent among patients than controls. Mood disorders in $4 \%$ and anxiety disorders in $2 \%$ were not significantly different from control prevalences. (Mannuzza S, Klein RG, Bessler A, Malloy P, LaPadula M. Adult psychiatric status of hyperactive boys grown up. Am I Psychiatry April 1998;155:493-498). (Respond: Dr Salvatore Mannuzza, Department of Clinical Psychology, New York State Psychiatric Institute, 722 West 168th St, New York, NY 10032).

COMMENT. Children with uncomplicated ADHD referred to a psychiatric clinic may "outgrow" the symptoms of hyperactivity and inattentiveness but they are at risk of developing antisocial pers nnality and nonalcoholic substance abuse disorders as grown ups. Substance abuse disorders in adults with a history of ADHD have been reported previously, but in association with persistence of ADHD.

Shaffer D, also from the NY State Psychiatric Institute, reported in 1994 that psychoactive substance abuse disorder is commonly associated with diagnoses of ADHD in adults. Biederman J et al, at the Massachusetts General Hospital, found in 1995 that childhood onset ADHD persisting in adults without comorbidity carried a $40 \%$ risk of substance abuse disorders, most commonly marijuana. Risk factors for persistence of ADHD into adolescence included 1) a genetic familiality of ADHD, 2) environmental psychosocial adversity and exposure to parental conflict, and 3) comorbidity with conduct, mood and anxiety disorders. (See PPN III, 1997; pp 231233).

The probands in the NY study did not suffer from the comorbid conduct disorders, common to $30 \%-50 \%$ of children with ADHD seen at psychiatric centers. Follow-up studies of children with ADHD referred to pediatric neurologists would be of interest. Selection of cases based on clinic referral may be important in outcome epidemiological studies of ADHD.

\title{
STIMULANT INDUCED TRICHOTILLOMANIA IN ADHD
}

The onset or exacerbation of trichotillomania (TT) within 6 months of starting treatment with methylphenidate (dose unreported) for ADHD is reported in 3 boys, aged 7 to 12 years, followed at the Yale Child Study Center, New Haven, CT. All three reported subjective tension relieved after hair-pulling from the scalp, and one also pulled and ate his eyebrows and eyelashes. Symptoms abated slowly or diminished in two, despite continuation of MPH; TT persisted in one after switching to imipramine. In a clinic for TT, affected boys had greater withdrawal and social problems than girls. Boys were more likely to suffer from ADHD and to be exposed to stimulant therapy. (Martin A, Scahill L, Vitulano L, King RA. Stimulant use and trichotillomania. I Am Acad Child Adolesc Psychiatry April 1998;37:349-350). (Respond: Andres Martin MD, Yale Child Study Center, New Haven, CT).

COMMENT. Tics and compulsive behaviors are well known complications of stimulant therapy for ADHD. Trichotillomania, a further side-effect of MPH to be aware of, has been seen occasionally in our own clinic for ADHD where relatively low doses of MPH are usually prescribed.

Retrospective review of treatment outcome for 63 patients with trichotillomania is reported from the Department of Psychiatry, Massachusetts General Hospital, Charlestown, MA 02129. (Keuthen NJ, O'Sullivan RL, Goodchild P et al. Am I Psychiatry April 1998;155:560-561). Mean age at onset was 12 years and age at follow up was 33 years. The patients ( $92 \%$ female, $8 \%$ male) had been treated for 6 years: $57(90 \%)$ received behavioral treatment, $46(73 \%)$ medication, and 41 
(65\%) both medication and behavioral therapy. Various medications were prescribed, including clomipramine (59\%), fluoxetine (50\%), and lithium (15\%). At follow-up, 32 (51\%) were still receiving some therapy, and 27 (49\%) were untreated. Total Hairpulling Scale scores showed significant improvements in $52 \%$, and no response in $48 \%$. Improvements had also occurred in measurements of self-esteem, psychosocial functioning, depression and anxiety. Responders had higher baseline depression scores than nonresponders, but a cause and effect correlation was undetermined. None of these subjects had ADHD and none received stimulant therapy.

\section{BUSPIRONE IN ADHD}

Buspirone monotherapy $(0.5 \mathrm{mg} / \mathrm{kg}$ daily for 6 weeks) was evaluated in 12 children (10 boys, 2 girls), aged 6 to 12 years, with ADHD at the Maudsley Hospital, London, UK. Compared to baseline scores on Conners Parent and other rating scales, all 12 patients showed significant improvements in hyperacti ity, impulsivity, inattention, and disruptive behavior. Behaviors resumed on discontinuing Buspirone after 6 weeks. Mild dizziness occurred in 2 in the first week. (Malhotra S, Santosh PJ. An open clinical trial of buspirone in children with attention-deficit/hyperactivity disorder. I Am Acad Child Adolesc Psychiatry April 1998;37:364-371). (Reprints: Dr Santosh, Child and Adoilescent Psychiatry, Maudsley Hospital, Denmark Hill, London SE5 8AZ, England).

COMMENT. Controlled trials of buspirone in larger samples of ADHD children seem justified. Buspirone is reported to increase noradrener ic and dopaminergic output, while modulating the serotonergic system. Used previously in children with anxiety, buspirone was tried in ADHD because of low toxicity and twice-daily regimen, not interfering with school schedules. All four dimensions of attention, hyperactivity, impulsivity, and behavioral problems improved, without rebound worsening of symptoms or development of tolerance. Effects on cognitive function need to be addressed, using psychological testing and teacher rating scales.

\section{THE MOZART SONATA AND SPATIAL-TEMPORAL REASONING}

The positive effect of music on spatial-temporal reasoning has been

demonstrated in college students using coherence analysis of electroencephalographic (EEG) recordings at the Center for Neurobiology of Learning and Memory and Department of Physics, University of California, Irvine CA. Right frontal and left temporo-parietal coherent EEG activity was induced by listening to 10 minutes of Mozart (Sonata for Two Pianos in D Major), and enhancement of spatial-temporal reasoning was carried over in 3 of 7 subjects. The long-lasting coherent EEG pattern might indicate structured sequences occurring in cortical function and extending over minutes. (Sarnthein J, vonStein A, Rappelsberger P et al. Pe sistent patterns of brain activity: An EEG coherence study of the positive effect of music on spatial-temporal reasoning. Neurol Res April 1998;19:107-116). (Reprints: Dr Gordon Shaw, Center for Neurobiology of Learning and Memory and Departemt of Physics, University of California, Irvine CA 92697).

COMMENT. Spatial-temporal reasoning is required for chess, mathematics and music appreciation. Exposure to music, specifically Mozart, has been found to enhance cortical activity used in spatial-temporal reasoning, whereas relaxation tapes and minimalist music had no effect. EEG coherence correlates to complex higher brain function, and patterns of cortical activity induced by listening to Mozart carry over to tasks involving spatial-temporal reasoning. 